\title{
LAS EDUSI PUESTAS EN VALOR: UNA MIRADA DESDE EL DERECHO ADMINISTRATIVO Y EL URBANISMO
}

\author{
María Luisa Gómez Jiménez \\ Directora WPS- RISHUR \\ mlgomez@uma.es
}

\begin{abstract}
El Desarrollo Urbano Sostenible, sigue siendo a pesar del tiempo que ha transcurrido desde su inicial formulación, uno de los retos a alcanzar en las políticas públicas que se desarrollan en el ámbito urbanístico en nuestras ciudades. El número monográfico que tiene entre sus manos obedece al examen de un aspecto destacado del mismo formulado desde la premisa de lo que se ha venido en llamar un desarrollo sostenible e integrado. Cuestión que aboca a los parámetros de gobernanza y buen gobierno, y que permite dibujar el camino de las estregadas de actuación más que de las directrices o regulaciones sectoriales detalladas. En todo caso porque la definición y el cumplimiento de las correspondientes estrategias va llevar aparejada la obtención también de financiación estratégica que finalmente - en tanto que medida de fomentoacaba siendo el leit motiv de muchas de las actuaciones que se han desarrollado en el espacio urbano de nuestras ciudades a tenor de las denominadas EDUSI, o estrategias de Desarrollo Urbano Sostenible.
\end{abstract}

Dentro de la Red Temática de Excelencia del Ministerio URBANRED, el estudio y la atención a las EDUSI ha sido un elemento de significativa importancia para la configuración de cuáles son las líneas por las que debiera discurrir en los años venideros las políticas públicas urbanística e inmobiliarias, y que grado de atención han tenido estas en su implementación en el territorio español.

En este sentido, el número que presentamos en esta ocasión incorpora estudios centrados en el examen de las Estrategias de Desarrollo Urbano Sostenible, tanto desde la formulación de los parámetros de sostenibilidad que incorporan, como desde la proyección del examen de casos concretos que se han examinado como

WPS RI-SHUR, n6, 2017, vol.2, ISSN: 2387-1768

MONOGRÁFICO SOBRE LAS ESTRATEGIAS DE DESARROLLO URBANO

SOSTENIBLE E INTEGRADO (EDUSI) 


\section{WPSReview International on Sustainable}

\section{Housing and Urban Renewal (RI-SHUR)}

propuestas de actuación. Así, se lleva a cabo un examen detallado de las herramientas e instrumentos de financiación que desde instancias comunitarias se han orientado a tal fin.

Este número se inicia con la incorporación de tres trabajos relacionados con la proyección de las EDUSIS y el desarrollo urbano sostenible. El primero, de la profesora. Alonso Ibañez, catedrática de Derecho Administrativo de la Universidad de Oviedo y directora del Proyecto de Red Temática de Excelencia URBANRED, analiza los elementos clave de la financiación comunitaria del Desarrollo Urbano, y nos recuerda la necesidad de aplicar enfoque integrados en la política de cohesión. Enfoques integrados que deben además - señala la autora- quedar garantizados mediante la participación local, y que deben ser puestos en la perspectiva del Programa Operativo de Crecimiento Sostenible, 2014-2020.

Las clarificadoras afirmaciones que contienen esta primera presentación, dan paso a la contribución del profesor Dr. García Rubio, titular de Derecho Administrativo de la Universidad Rey Juan Carlos, en torno al examen de los modelos de desarrollo urbanos sostenible en Iberoamérica. El examen del Derecho Urbanístico comparado, incorpora en este caso el examen del ordenamiento jurídico español, el de Colombia, la República Dominicana y Uruguay. El examen de países latinoamericano se enriquece además con una proyección necesaria de las políticas públicas urbanísticas y medidas que abocan a un desarrollo sostenible presente pero en diferente grado de ejecución en todas ellas.

El acierto del profesor García Rubio, radica no sólo en la original de su aporte sino en contrastar que las premisas si bien pueden ser cambiantes abocan a resultados comunes en los países examinados. Así las cosas, la proyección de derecho comparado, nos devuelve al escenario europeo, y al régimen de las EDUSI en el Derecho Comunitario, cuestión que es abordada desde la proyección de las mismas en el Derecho Español. Visión que incorpora el trabajo de las profesoras Dr. De Gregorio Hurtado, y González Medina, ambas profesoras de la Universidad Politécnica de Madrid, que nos avanzan de las Ciencias Políticas una precisa valoración de la proyección de las EDUSI en España, para ello nada mejor que llevar a cabo una revisión de las iniciativas Comunitarias, y los programas que han permitido la calificación de las dinámicas actuales de las Estrategias de Desarrollo Urbanos

WPS RI-SHUR, n6, 2017, vol.2, ISSN: 2387-1768

MONOGRÁFICO SOBRE LAS ESTRATEGIAS DE DESARROLLO URBANO SOSTENIBLE E INTEGRADO (EDUSI) 


\section{WPSReview International on Sustainable \\ Housing and Urban Renewal (RI-SHUR)}

Integrado. El brillante aporte de las profesoras evidencia eso sí, el impacto que las políticas comunitarias tienen en la configuración de desarrollo urbano sostenible en nuestro país.

Así, las cosas, prosigue el monográfico que les traemos con el examen de casos concretos de aplicación y desarrollos de las EDUSI en la geografía española, en concreto se aportan en la segunda sección, relativa a las propuestas de actuación, tres estudios de casos, a saber: el correspondiente a las EDUSI en Aragón, en Asturias, y en Cataluña.

Somos conscientes de que hubiera sido preciso integrar una mayor proyección territorial, cuestión que dejamos para un momento posterior siendo en cierta forma los tres casos - que traemos en esta ocasión, emblemáticos y representativos.

El primero corre a cargo de la profesora Carmen de Guerrero, profesora de Derecho Administrativo de la Universidad de Zaragoza, perteneciente al grupo de Investigación ADESTER (Administración, Economía, Sociedad y Territorio). La contribución de la Dra. De Guerrero analiza en inglés la aplicación de las EDUSI en Aragón, y lo hace destacando los aspectos de financiación que se incorporan a partir de las previsiones comunitarias y en el marco del Programa Operativo 2014-2020, así como las dificultades para su implementación desde la perspectiva del acceso a dicha financiación y sus efectos en el territorio.

El segundo estudio de casos, contempla en secuencia de continuidad, la aplicación de las EDUSI en el caso de Asturias. Nadie mejor que la profesora Dra. María Cruz Rozada Ardavin, de la Universidad de Oviedo que desarrolla su actividad en el Grupo de Trabajo de Desarrollo Sostenible (GDTS), para llevar a cabo dicha tarea.

Así, el estudio que nos presenta la profesora Rozada Ardavín, que examina el caso contrapuesto de Mieres, y Avilés, con la idea de aproximar el grado de desarrollo de las EDUSI, en la Comunidad Autónoma. Se trata en suma del examen de dos respuestas por ciudades que han recibido financiación a través de las EDUSI, siendo que se operan significativos contrastes y desarrollos de sumo interés para el lector.

Finalmente, cierra el número la contribución el Dr. Albert Albert Lladó Martínez, profesor de Derecho Administrativo de la Universidad de Girona y miembro del Grupo

WPS RI-SHUR, n6, 2017, vol.2, ISSN: 2387-1768

MONOGRÁFICO SOBRE LAS ESTRATEGIAS DE DESARROLLO URBANO SOSTENIBLE E INTEGRADO (EDUSI) 


\section{WPSReview International on Sustainable \\ Housing and Urban Renewal (RI-SHUR)}

de Investigación Grup de Recerca de Dret Urbanístic, que se centra en el examen de las EDUSI en Cataluña. La investigación que el profesor Lladó aúna el interés de las Convocatorias 2015 y 2016, con la maestría con la que el autor desgrana la proyección de las mismas en los municipios catalanes que resultaron beneficiarios de las ayudas.

Dibujan pues los tres casos presentados un escenario dispar que sin embargo no ha terminado de definirse, al albur de las líneas de financiación previstas en los próximo años.

Resulta no obstante llamativo que la temática que en esta ocasión traemos a WPS RISHUR, no haya sido profusamente examinada por la doctrina, ni en general conocida por el profano, incluso dedicado a cuestiones urbanas, lo cual de alguna forma refleja el distanciamiento real de la proyección europea en las esferas regionales, o locales en la que se proyectan políticas y dinámicas necesitadas de esquemas operativos y funcionales congruentes con la misma configuración del sistema urbanístico Español .

De ahí, aún más si cabe, el interés de esta monográfico que ha sido auspiciado por la Red Temática de Excelencia del Ministerio, sobre desarrollo Urbano Sostenible (URBAN RED) del Programa Estatal de Investigación Científica y Técnica de Excelencia, Subprograma Estatal de Generación de Conocimiento, en el marco del Plan Estatal de Investigación Científica y Técnica y de Innovación 2013-2016 (Proyecto: DER2015-71345-REDT), dentro del Seminario de Trabajo sobre las EDUSI que en el seno de URBANRED, tuvo lugar el pasado 13 de octubre de 2017, en la sede del Seminario García de Enterría, de la Facultad de Derecho de la Universidad Complutense de Madrid, y del que damos cuenta en el presente volumen, confiando colme sus expectativas.

En Málaga, a 30 de diciembre de 2017

WPS RI-SHUR, n6, 2017, vol.2, ISSN: 2387-1768

MONOGRÁFICO SOBRE LAS ESTRATEGIAS DE DESARROLLO URBANO

SOSTENIBLE E INTEGRADO (EDUSI) 